Claremont Colleges

Scholarship@ Claremont

All HMC Faculty Publications and Research

HMC Faculty Scholarship

$11-1-1968$

\title{
A Theoretical Model for Gas Separation in a Glow Discharge: Cataphoresis
}

Fredrick H. Shair

California Institute of Technology

Donald S. Remer

Harvey Mudd College

\section{Recommended Citation}

Shair, Fredrick H., and Donald S. Remer. "A Theoretical Model for Gas Separation in a Glow Discharge: Cataphoresis." Journal of Applied Physics 39.12 (1968): 5762-5767. DOI: 10.1063/1.1656046

This Article is brought to you for free and open access by the HMC Faculty Scholarship at Scholarship @ Claremont. It has been accepted for inclusion in All HMC Faculty Publications and Research by an authorized administrator of Scholarship @ Claremont. For more information, please contact scholarship@cuc.claremont.edu. 


\title{
A Theoretical Model for Gas Separation in a Glow Discharge: Cataphoresis
}

\author{
Fredrick H. Shatr and Donald S. Remer \\ Division of Chemistry and Chemical Engineering, California Institute of Technology, Pasadena, California 91109
}

(Received 4 December 1967; in final form 29 July 1968)

\begin{abstract}
A theoretical model for transient and steady-state cataphoresis is developed starting with the macroscopic equations of continuity. After a brief breakdown period, the impurity ions are assumed to be closely coupled with their neutral counterparts. The basic assumptions in the model are that after breakdown, the level of ionization of the impurity, and the axial electric field remain constant; it is demonstrated that under these conditions a system involving rapid ionization-recombination reactions is equivalent to a system in which no reaction occurs, but in which the "effective" ion mobility is a product of the true ion mobility and the fraction of impurity ionization. The influence of endbulbs commonly employed in experiments is analyzed and found to influence greatly the characteristic time required to reach steady state. Agreement is found between the model and available experimental data. Particular emphasis is placed upon mass spectrometer data reported by Matveeva, and by Beckey, Groth, and Welge; these data are for mixtures of rare gases and for mixtures of hydrogen and deuterium, and involve endbulbs. The ordinary diffusion case, associated with the collapse of the steady-state cataphoretic profile, is also analyzed for a system containing endbulbs.
\end{abstract}

\section{INTRODUCTION}

It has been known for some time that longitudinal concentration gradients occurred within dc glowdischarge tubes containing various gas mixtures. ${ }^{1}$ This effect, resulting from one component being drawn preferentially toward the cathode, has been termed cataphoresis. ${ }^{2}$ This phenomenon has been known to occur in at least 23 different binary mixtures. ${ }^{1-14}$ The work up to 1958, involving rare gases, was reviewed by Loeb. ${ }^{15}$ Cataphoresis has been used primarily as a convenient technique to provide pure gases ${ }^{16,17}$; occasionally, cataphoresis has been used to obtain an impurity level of one part in $10^{7}$ or less. ${ }^{9,10}$ As pointed out by Oskam, ${ }^{18}$ "even the application of the most refined ultrahigh vacuum techniques cannot remove the rare gas impurities in commercially available gases."19 Consequently, it is of interest to obtain a better description on both the microscopic and macroscopic levels. Concerning the separation of isotopes, Groth and Harteck $^{13}$ found an enrichment of deuterium at the cathode in the case of hydrogen. Continuing along this

\footnotetext{
1 E. C. C. Baly, Phil. Mag 35, 200 (1893).

2 G. Francis, Encyclopedia of Physics, XXII, Gas Discharges II, (Springer-Verlag, Berlin, 1956), pl 195.

${ }^{3}$ J. J. Thompson, Proc. Roy. Soc. (London) 58, 244 (1895).

4 F. Skaupy, Verh. Deut. Phys. Ges. $\langle 8,230$ (1916).

${ }^{5}$ F. Skaupy and F. Bobek, Z. Tech. Physik 6, 284 (1925).

- F. M. Penning, Physica 1, 763 (1933).

${ }^{7}$ M. J. Druyvesteyn and N. Warmoltz, Phil. Mag. 17, 1, (1934).

${ }^{8}$ M. J. Druyvesteyn, Physica 2, 255 (1935).

${ }^{9}$ R. Riesz and G. H. Dieke, J. Appl. Phys. 25, 196 (1954).

${ }^{10}$ H. C. Miller, General Electric Research Rept. No. 63-RL3462G (1963).

${ }^{11}$ A. L. Schmeltekopf, Jr., J. Appl. Phys. 35, 1712 (1964).

${ }_{12}$ N. A. Matveeva, Bull. Acad. Sci. USSR Phys. Ser. (English Ed.) 23, 1009 (1959).

13 W. Groth and P. Harteck, Naturwiss. 22, 391 (1939).

${ }^{14} \mathrm{H}$. D. Beckey, W. E. Groth, and K. H. Welge, Z. Naturforsch. 8a, 556 (1953).

${ }_{15}$ L. B. Loeb, J. Appl. Phys. 29, 1369 (1958) .

${ }^{16}$ V. R. Mittelstadt and H. J. Oskam, Rev. Sci. Instr. 32, 1408 (1961).

${ }^{17}$ L. B. Loeb, R. G. Westberg, and H. C. Huang, Phys. Rev. 123,43 (1961).

${ }_{18}$ H. J. Oskam, J. Appl. Phys. 34, 711 (1963).

19 The italics are Oskam's (Ref. 18).
}

line, Beckey, Groth, and Welge ${ }^{14}$ reported experimental results in which a mass spectrometer was used to monitor the concentration of deuterium and hydrogen in the cathode and anode regions. Later, Matveeva ${ }^{12}$ reported mass spectrometer data which she obtained during studies involving binary mixtures of $\mathrm{He}, \mathrm{Ar}$, and Ne. Often, the electrodes have been placed within bulbs which are located at the ends of the discharge tube. ${ }^{9,12,14}$

Druyvesteyn ${ }^{8}$ presented an approximate treatment for a steady-state cataphoresis without endbulbs. Recently, Freudenthal $1^{20,21}$ has developed a linearized model of transient cataphoresis when no endbulbs are present. Presented below is a linearized macroscopic model of transient cataphoresis when endbulbs are present. ${ }^{22}$ Quantitative comparison is made between the model and the mass spectrometer data reported by Beckey, Groth, and Welge, ${ }^{14}$ and by Matveeva. ${ }^{12}$ The mass spectrometer data were favored because (1) the initial impurity compositions were reported, and (2) the problem associated with the optical spectroscopic technique near the cathode ${ }^{11}$ was avoided. Due to the lack of completeness associated with the optical spectroscopic data reported previously, only a qualitative comparison was made between these data and the model.

Using a system containing endbulbs, Matveeva ${ }^{12}$ also studied the collapse of the steady-state cataphoretic profile, after the electrical discharge was extinguished. It is mentioned that Hogervorst and Freudenthal ${ }^{23}$ recently used this system without endbulbs to measure binary diffusion coefficients.

\section{ANALYSIS}

\section{Cataphoresis}

The mass conservation equations are applied to the impurity ions and to the impurity neutrals, immediately

${ }^{20}$ J. Freudenthal, Physica 36, 354 (1967).

21 J. Freudenthal, J. Appl. Phys. 38, 4818 (1967).

22 This research commenced independently of that reported by Freudenthal ${ }^{20,21}$ and by Hogervorst and Freudenthal. ${ }^{23}$

${ }^{23} \mathrm{~W}$. Hogervorst and J. Freudenthal, Physica 37, 97 (1967) 
after the electric discharge is established. Multiple ionization processes are neglected. Both the electric field, and the ratio of impurity ion concentration to impurity neutral concentration are taken to be constant. When endbulbs are present, the concentration throughout each endbulb is taken to be uniform and equal to the value of the composition at the nearest end of the discharge tube. Only electron impact ionization processes are considered. Whereas the ionization process is taken to be homogeneous in character, the main loss of charged particles is considered to be through ambipolar diffusion to the walls where recombination occurs; this has been the starting point in the development of other theories concerning the positive column. ${ }^{2,24,25}$ Let the cathode be situated at $z=0$. The starting equation for the impurity ions is

$$
\begin{array}{r}
\partial n_{+} / \partial t=D_{+}\left(\partial^{2} n_{+} / \partial Z^{2}\right)+D_{a}(1 / r)(\partial / \partial r)\left[r\left(\partial n_{+} / \partial r\right)\right] \\
+\mu E\left(\partial n_{+} / \partial Z\right)+R_{i} .
\end{array}
$$

The starting equation for the impurity neutrals is $\partial n_{0} / \partial t=D\left(\partial^{2} n_{0} / \partial Z^{2}\right)+D(1 / r)(\partial / \partial r)\left[r\left(\partial n_{0} / \partial r\right)\right]-R_{i}$.

The first term on the right-hand side represents the ordinary diffusion in the direction parallel to the axis of the tube. We have assumed the diffusion coefficients to be independent of composition. The second term represents the radial diffusion of ions towards the wall, and the radial diffusion of neutrals away from the wall. The third term in the ion equation represents forced diffusion due to a uniform axial electric field. The last term represents the rate of homogeneous ionization which is a source for ions and a sink for neutrals. The quantities $D, D_{+}$, and $D_{a}$ are the diffusion coefficients for neutral diffusion, ion diffusion, and ambipolar diffusion, respectively. As a first approximation, we shall treat the problem assuming $D \simeq D_{+}$. The nondimensional diffusion equations then become

$$
\begin{aligned}
\partial C_{+} / \partial \tau= & \left(\partial^{2} C_{+} / \partial \eta^{2}\right) \\
& +\left(L^{2} D_{a} / R^{2} D_{\rho}\right)(\partial / \partial \rho)\left[\rho\left(\partial C_{+} / \partial \rho\right)\right] \\
& +\left[\beta\left(\partial C_{+} / \partial \eta\right)\right]+\left(L^{2} / D\right) R_{i}
\end{aligned}
$$

and

$$
\begin{aligned}
\partial C_{0} / \partial \tau & =\left(\partial^{2} C_{0} / \partial \eta^{2}\right) \\
+ & \left(L^{2} / R^{2} \rho\right)(\partial / \partial \rho)\left[\rho\left(\partial C_{0} / \partial \rho\right)\right]-\left(L^{2} / D\right) R_{i},
\end{aligned}
$$

where

$\tau=t D / L^{2}, \quad \eta=Z / L, \quad \rho=r / R$,

$$
\beta=\mu E L / D, \quad C_{+}=n_{+} / n_{0}{ }^{0}
$$

and $C_{0}=n_{0} / n_{0}^{0}$. Here $L$ represents the tube length, $R$ the tube radius, and $n_{0}{ }^{0}$ is the density of the impurity

${ }^{24}$ F. Llewelleyn-Jones, Methuen \& Co., Ltd. (London) 1966.

${ }^{25}$ I. M. Cohen and M. D. Kruskal, Phys. Fluids 8, 920 (1965). which is uniformly dispersed throughout the system before breakdown. The quantity $\beta$ represents the ratio of the forced diffusion effect to the ordinary diffusion effect.

Multiplying Eqs. (3) and (4) by $\rho$ and integrating from the tube center at $\rho=0$ to the wall at $\rho=1$ yields

$$
\begin{array}{r}
\partial\left\langle C_{+}\right\rangle / \partial \tau=\left(\partial^{2}\left\langle C_{+}\right\rangle / \partial \eta^{2}\right)+\left(L^{2} D_{a} / R^{2} D\right)\left(\partial C_{+} / \partial \rho\right)_{\rho=1} \\
+\beta\left(\partial\left\langle C_{+}\right\rangle / \partial \eta\right)+\left(L^{2} / D\right)\left\langle R_{i}\right\rangle
\end{array}
$$

and

$$
\begin{aligned}
\partial\left\langle C_{0}\right\rangle / \partial \tau=\partial^{2}\left\langle C_{0}\right\rangle / \partial \eta^{2}+\left(L^{2} / R^{2}\right)\left(\partial C_{0} / \partial \rho\right)_{\rho=1} \\
-\left(L^{2} / D\right)\left\langle R_{i}\right\rangle .
\end{aligned}
$$

The brackets indicate radially averaged quantities such as

$$
\left\langle C_{+}\right\rangle=\int_{0}^{1} \rho C_{+}(\rho) d \rho
$$

All ions diffusing to the wall are assumed to be lost, and to recombine rapidly as compared to the characteristic times associated with the diffusion processes. For small diameter tubes, the radial diffusion terms are much larger than the longitudinal diffusion terms. Thus,

$$
D_{a}\left(\partial C_{+} / \partial \rho\right)_{\rho=1}=-D\left(\partial C_{0} / \partial \rho\right)_{\rho=1} .
$$

Since $D_{a} \simeq D_{+}\left[1+\left(T_{e} / T_{i}\right)\right]$ and $T_{e} \gg T_{i}$ in the positive column of the glow discharge, then $\left|\left(\partial C_{+} / \partial_{\rho}\right)_{\rho=1}\right| \ll$ $\left|\left(\partial C_{0} / \partial \rho\right)_{\rho=1}\right|$.

The level of ionization of the impurity,

$$
\left\langle C_{+}\right\rangle /\left(\left\langle C_{+}\right\rangle+\left\langle C_{0}\right\rangle\right),
$$

is assumed constant and independent of time and of position in the discharge. This assumption implies that we do not treat the case where the ionization frequencies of the impurity and of the host gas are of the same order. Adding Eqs. (5) and (6) and rearranging yields a general equation for cataphoresis:

$$
\partial \theta / \partial \tau=\left(\partial^{2} \theta / \partial \eta^{2}\right)+\alpha(\partial \theta / \partial \eta)
$$

We have defined

and

$$
\begin{gathered}
\theta=\left(\left\langle C_{0}\right\rangle+\left\langle C_{+}\right\rangle\right) \\
\alpha=\left(\left\langle C_{+}\right\rangle /\left\langle C_{0}\right\rangle+\left\langle C_{+}\right\rangle\right)(\mu E L / D) .
\end{gathered}
$$

Thus, when the impurity ions and neutrals are tightly coupled, the reacting system is found to be equivalent to an inert system; however, the inert system is one in which the "effective" ion mobility is a product of the true ion mobility and the level of ionization of the impurity. When $\left\langle C_{+}\right\rangle\left\langle\left\langle C_{0}\right\rangle\right.$, then $\alpha \sim\left(\left\langle C_{+}\right\rangle /\left\langle C_{0}\right\rangle\right)(\mu E L / D)$ and Eq. (9) reduces to Freudenthal's principle equation..$^{20,21}$

Equation (9) must be solved under appropriate boundary conditions for the experiments considered. The boundary conditions which represent the influence of the endbulbs are

$$
(\partial \theta / \partial \eta)+\alpha \theta=\delta(\partial \theta / \partial \tau) \quad \text { at } \eta=0
$$




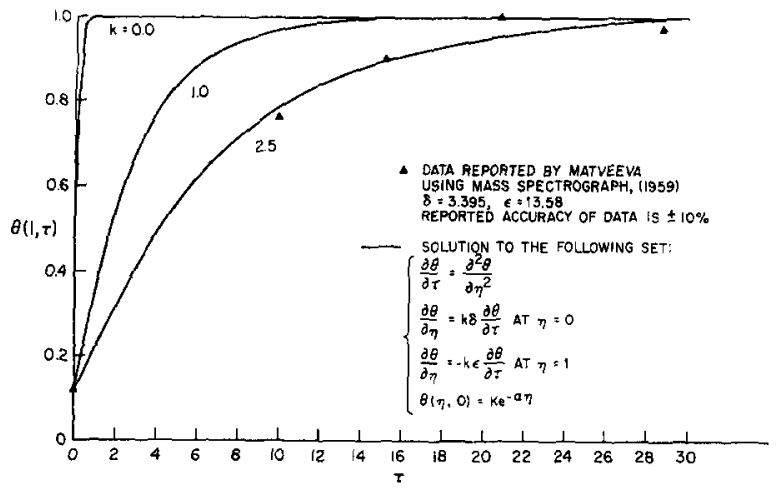

FIG. 1. Argon concentration vs time near the anode for backward diffusion in argon-helium mixture.

and

$$
(\partial \theta / \partial \eta)+\alpha \theta=-\epsilon(\partial \theta / \partial \tau) \quad \text { at } \eta=1 .
$$

The quantity $\delta$ is the ratio of the volume of the bulb surrounding the cathode to the volume of the discharge tube. Similarly, $\epsilon$, is the ratio of the volume of the bulb surrounding the anode to the volume of the discharge tube. Equation (10) states that a flux of impurity out of the tube at $\eta=0$ results in an increase in the concentration of impurity in the bulb surrounding the cathode.

On the other hand, Eq. (11) states that a flux of impurity into the tube at $\eta=1$ results in a decrease in the concentration of impurity in the bulb surrounding the anode. Each endbulb is assumed to be well mixed so that the impurity composition in the cathode bulb is uniform and equal to the impurity composition at $\eta=0$; likewise, the impurity composition in the anode bulb is assumed uniform and equal to the impurity composition at $\eta=1$.

The initial condition is

$$
\theta=1 \quad \text { for all } \eta \text {. }
$$

It should be noted that the eigenfunctions for the above problem are not orthogonal in the usual sense. Since the problem has been solved elsewhere, ${ }^{26}$ we shall merely write down the solution:

$$
\begin{aligned}
& \theta(\eta, \tau)= K e^{-\alpha \eta}+\alpha e^{-\alpha \eta / 2} \exp \left(-\alpha^{2} \tau / 4\right) \sum_{n=1}^{\infty} A_{n}{ }^{-1} \\
& \times\left\{\left[\mu_{n} \cos \mu_{n}(1-\eta)+E_{n} \sin \mu_{n}(1-\eta)\right]\right. \\
&\left.-e^{\alpha / 2}\left[\mu_{n} \cos \mu_{n} \eta-D_{n} \sin \mu_{n} \eta\right]\right\} \exp \left(-\mu_{n}{ }^{2} \tau\right),
\end{aligned}
$$

where

$$
\begin{aligned}
& A_{n}=\left[\left(\alpha^{2} / 4\right)+\mu_{n}{ }^{2}\right]^{2}\left\{[\delta \epsilon+(\delta+\epsilon) / 2] \sin \mu_{n}\right. \\
& \left.-F_{n}\left(\cos \mu_{n} / 2 \mu_{n}\right)\right\} \\
& D_{n}=\frac{1}{2} \alpha\left[1+\delta\left(\frac{1}{2} \alpha\right)\right]+\delta \mu_{n}^{2} \\
& E_{n}=\frac{1}{2} \alpha\left[1-\epsilon\left(\frac{1}{2} \alpha\right)\right]-\epsilon \mu_{n}^{2} \\
& F_{n}=1+\delta+\epsilon+(\alpha / 2)(\delta-\epsilon)-\delta \epsilon\left(\frac{1}{4} \alpha^{2}+\mu_{n}^{2}\right)
\end{aligned}
$$

and

$$
K=(1+\delta+\epsilon) /\left(\delta+\epsilon e^{-\alpha}-e^{-\alpha} / \alpha+1 / \alpha\right) .
$$

The terms, $\mu_{n}$, are solutions to the following equation:

$$
\tan \mu_{n}=-(\delta+\epsilon) \mu_{n} /\left[1+\frac{1}{2} \alpha(\delta-\epsilon)-\delta \epsilon\left(\frac{1}{4} \alpha^{2}+\mu_{n}^{2}\right)\right] \text {. }
$$

The first term in Eq. (13) represents the steady-state solution, while the rest represents the transient part which decays rapidly in time. The constant $K$ was obtained from a mass balance between the final and initial concentration profiles.

$$
\delta \theta(0, \infty)+\int_{0}^{1} K e^{-\alpha \eta} d \eta+\epsilon \theta(1, \infty)=1+\delta+\epsilon .
$$

Typical values of $\theta$ vs $\tau$ when $\delta=\epsilon$ are shown in Figs. 7 and 8.

\section{Collapse of the Steady-State Cataphoretic Profile}

At time equal to zero, the discharge is extinguished and the exponential concentration profile collapses in time to a flat uniform concentration profile. Here again, account is taken for the endbulbs present in the experiment. The equation to be solved is

$$
\partial \theta / \partial \tau=\partial^{2} \theta / \partial \eta^{2}
$$

with boundary conditions

and

$$
\partial \theta / \partial \eta=\delta(\partial \theta / \partial \tau) \quad \text { at } \eta=0
$$

$$
\partial \theta / \partial \eta=-\epsilon(\partial \theta / \partial \tau) \quad \text { at } \eta=1 .
$$

The initial condition for this case is

$$
\theta=K e^{-\alpha \eta}
$$

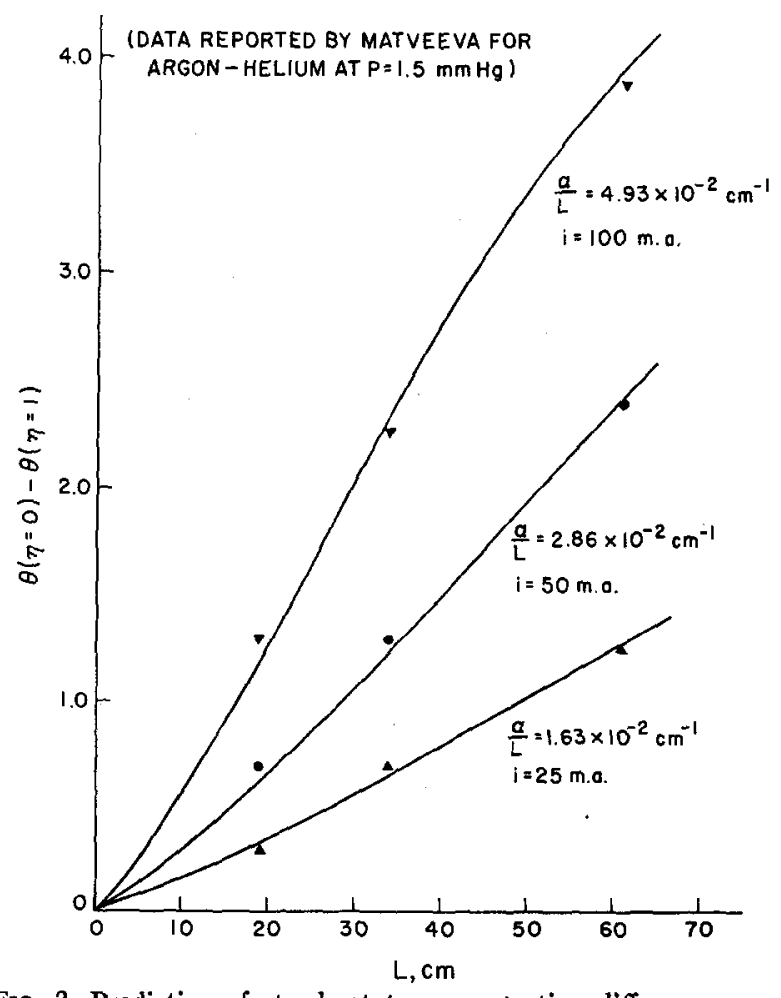

FIG. 2. Prediction of steady-state concentration difference as a function of $L$. 
As before, the problem is non self-adjoint and has been solved elsewhere. ${ }^{26}$ The solution is

$$
\begin{aligned}
& \theta(\eta, \tau)=1+2 \alpha K \sum_{n=1}^{\infty} B_{n}{ }^{-1}\left\{(1+\alpha \delta) B_{n}^{-1}\right. \\
& \times\left[\cos \mu_{n}(1-\eta)-\epsilon \mu_{n} \sin \mu_{n}(1-\eta)\right] \\
&\left.-(1-\alpha \epsilon)\left(\cos \mu_{n} \eta-\delta \mu_{n} \sin \mu_{n} \eta\right)\right\} \exp \left(-\mu_{n}^{2} \tau\right),
\end{aligned}
$$

where

$$
\begin{aligned}
B_{n}=\left(\alpha^{2}+\mu_{n}^{2}\right)[(1+\delta+\epsilon & \left.-\delta \epsilon \mu_{n}^{2}\right) \cos \mu_{n} \\
& \left.-(\delta+\epsilon+2 \delta \epsilon) \mu_{n} \sin \mu_{n}\right] .
\end{aligned}
$$

The terms $\mu_{n}$ for this problem are solutions to the following equation:

$$
\tan \mu_{n}=-(\delta+\epsilon) \mu_{n} /\left(1-\delta \epsilon \mu_{n}^{2}\right) .
$$

\section{DISCUSSION}

Calculations with Eq. (13) indicate that the steadystate cataphoretic profile is much more sensitive to $\alpha$ than to $\delta$ and $\epsilon$. On the other hand, the characteristic time $\tau_{c}$ associated with transient cataphoresis is quite insensitive to $\alpha$ but extremely sensitive to $\delta$ and $\epsilon$.

Matveeva ${ }^{12}$ monitored the composition near the anode during the buildup of the cataphoretic profile; in order to obtain agreement between the model and experiment an "effective" volume of each bulb, equal to 1.7 times that of the reported values, was required. This is not unexpected, ${ }^{27}$ since Eq. (13) is the solution to an idealized problem in which the endbulbs are adequately stirred. In Matveeva's ${ }^{12}$ transient cataphoretic experiment, it was found that $\tau_{c}=3.4$ and $\alpha=3.5$. Using the actual volumes of the endbulbs yields a $\tau_{c}=2.0$. When no endbulbs are present, the calculated $\tau_{c}=0.009$.

Matveeva ${ }^{12}$ also monitored the composition near the anode during the collapse of the cataphoretic profile. As indicated in Fig. 1, in order to obtain agreement

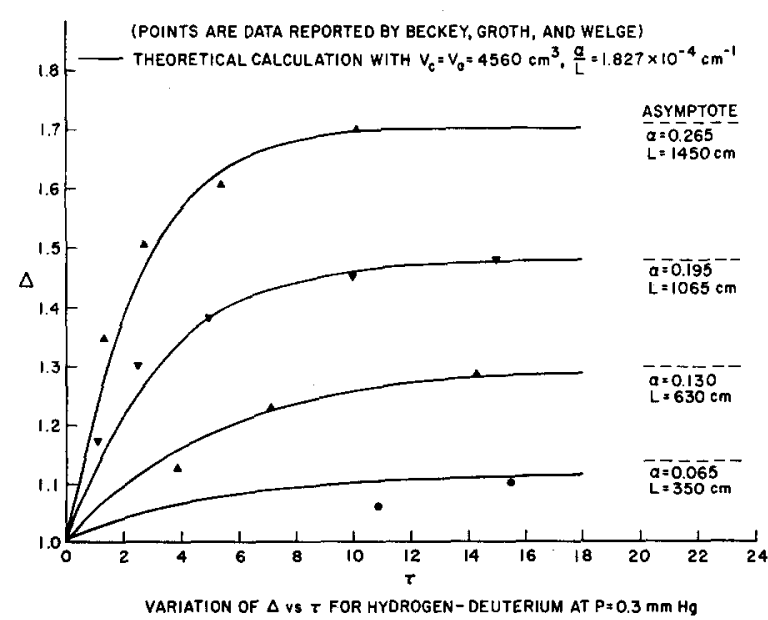

FIG. 3. Variation of $\Delta \mathrm{vs} \tau$ for hydrogen-deuterium at $P=0.3$ Torr.

${ }^{27}$ J. Crank, The Mathematics of Diffusion (Clarendon Press, Oxford, 1956).

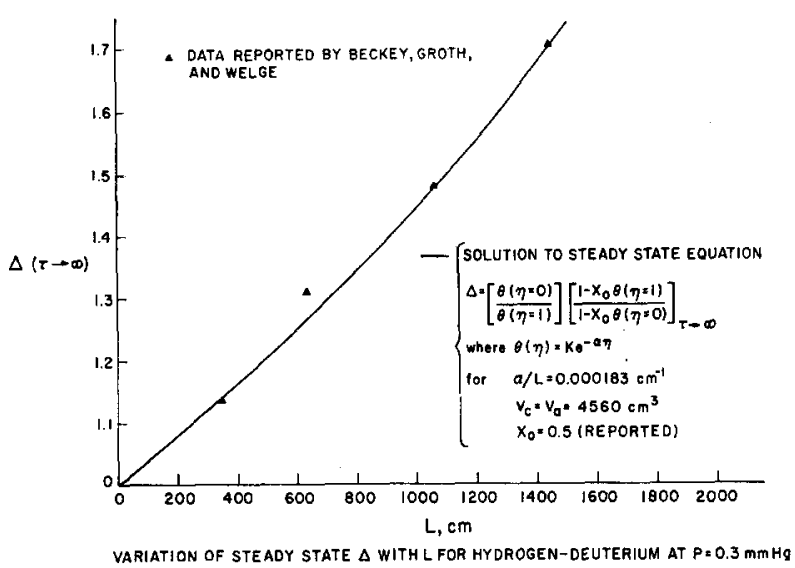

FrG. 4. Variation of steady-state $\Delta$ with $L$ for hydrogendeuterium at $P=0.3$ Torr.

between Eq. (25) and experiment, we had to take an "effective" volume of each bulb equal to 2.5 times that of the reported values. The difference in the effective volumes required for the two experiments may be due to some endbulb mixing in the cataphoretic experiment; electrode heating along with the discharge within the endbulbs, may have caused free convection.

The data reported in Matveeva's Figs. 3, 4, and 5 were then analyzed within the framework of our model. If $E \sim 10 \mathrm{~V} / \mathrm{cm}$, then calculated values of $\left\langle n_{+}\right\rangle$near the cathode region ranged from $10^{10}-10^{12}$ ions $/ \mathrm{cm}^{3}$; the corresponding values near the anode ranged from $10^{9}-10^{11}$ ions $/ \mathrm{cm}^{3}$. These values appear to be reasonable. It should be mentioned that the data points were measured by tracings onto $\mathrm{mm}$ graph paper. A comparison between our model and the data reported in Fig. 7 of Matveeva ${ }^{12}$ is shown in Fig. 2 of this text. For each of the three values of discharge currents, it was possible to obtain excellent agreement with a single value of $\alpha / L$.

Beckey, Groth, and Welge ${ }^{14}$ (referred to as BGW) monitored the concentration of $\mathrm{D}_{2}$ and $\mathrm{H}_{2}$ in both the anode and cathode regions. $\mathrm{BGW}$ reported their data using tau, which they defined

$$
\begin{aligned}
\tau_{\mathrm{BGW}} \equiv\left(\frac{\text { mole fraction of } \mathrm{D}_{2} \text { at cathode }}{\text { mole fraction of } \mathrm{D}_{2} \text { at anode }}\right) \\
\\
\quad \times\left(\frac{\text { mole fraction of } \mathrm{H}_{2} \text { at anode }}{\text { mole fraction of } \mathrm{H}_{2} \text { at cathode }}\right) .
\end{aligned}
$$

In order to avoid any ambiguity, we shall define $\Delta \equiv \tau_{\mathrm{BGW}}$, which is related to our $\theta$ by the following expression:

$$
\begin{aligned}
\Delta= & {[\theta(\eta=0, \tau) / \theta(\eta=1, \tau)] } \\
& \times\{[1-0.5 \theta(\eta=1, \tau)] /[1-0.5 \theta(\eta=0, \tau)]\} .
\end{aligned}
$$

In the above expression, $\tau$ refers to the dimensionless time as used in Eq. (13). The factor 0.5 is present because the initial mole fraction of deuterium was 0.5 in all the experiments reported. Unfortunately, BGW 


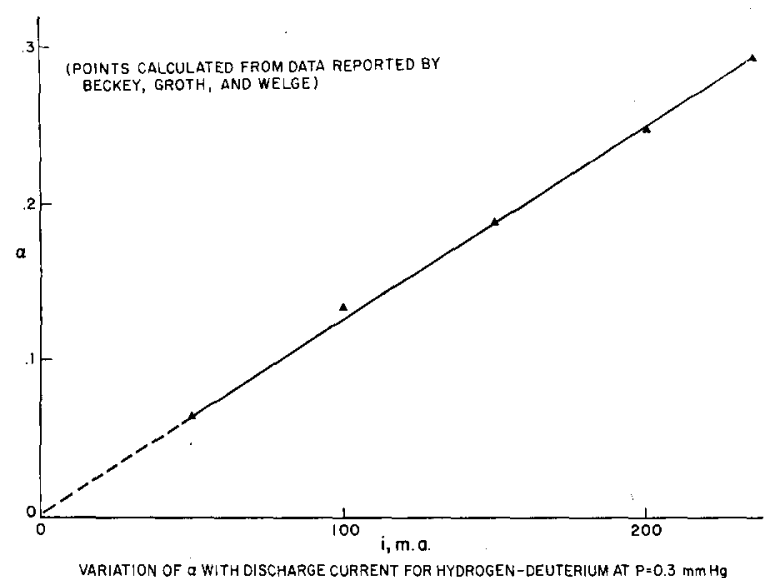

FIG. 5. Variation of $\alpha$ with discharge current for hydrogendeuterium at $P=0.3$ Torr.

report neither electric field data nor the volumes of the "large flasks situated near the anode and cathode." 28 However, from their Fig. 1, it is reasonable to assume that the volumes of both flasks are approximately equal, i.e., $\delta=\epsilon$.

As shown in Figs. 3 and 4, values of $V_{c}=V_{a}=4560$ $\mathrm{cm}^{3}$ and $\alpha / L=1.827 \times 10^{-4}$ allow agreement between our model and the data reported in Figs. 2 and 3 of $\mathrm{BGW}$. If $E \sim 10 \mathrm{~V} / \mathrm{cm}$, then calculated values of $\left\langle n_{+}\right\rangle$in the discharge ranged from $10^{10}$ to $10^{11}$ ions $/ \mathrm{cm}^{3}$. Figure 4 of $\mathrm{BGW}$ indicates that a plot of $\ln [\Delta(\tau \rightarrow \infty)-\Delta(\tau)]$ vs time is linear, which is in agreement with the model. Figure 5 of $\mathrm{BGW}$ indicates a linear relation between $\ln [\Delta(\tau \rightarrow \infty)]$ vs discharge current; as shown in our Fig. 5 , these data suggest that the quantity $\left(\left\langle n_{+}\right\rangle /\left\langle n_{0}\right\rangle\right) E$ is linearly proportional to the discharge current. This conclusion appears reasonable as a number of investigators ${ }^{29}$ have found that in the glow discharge the electron density at constant pressure is linearly proportional to the discharge current, while $E$ does not change much at higher currents.

Volume of Endbulbs: Equation (13) indicates that $\tau_{c}$ is linearly proportional to $\delta+\epsilon$. Thus, when $L$ and $R$ are kept constant, both $\tau_{c}$ and $t_{c}$ are linearly proportional to $V_{c}+V_{a}$. Riesz and Dieke ${ }^{9}$ reported: "The time at which equilibrium is reached depends on the volume of the bulb B. (This was a bulb attached to the anode region.) The larger its volume, the longer it takes before equilibrium is reached. If it is omitted equilibrium is reached within a minute; ...'"30 Undoubtedly, the long characteristic times reported by Matveeva ${ }^{12}$ and by $\mathrm{BGW}^{14}$ are due to the large values of $\delta$ and $\epsilon$ present in their experiments. The calculated influence of bulb size upon $\tau_{c}$ relevant to the data reported by

${ }^{28}$ The italics are BGW's (Ref. 14),

${ }^{29}$ Among others. J. F. Prince, and W. W. Robertson, J. Chem. Phys. 45, 2577 (1966).

${ }^{30}$ Yu A. Pekar, Sov. Phys. 11, 1024 (1967).
BGW, is shown in Fig. 6. As indicated in Fig. $6, \tau_{c}$ is relatively insensitive to $\alpha$.

A large endbulb at the cathode is predicted to decrease the steady-state concentration of the impurity near the anode; the limiting value of $\theta$ at $\eta=1$ is predicted to be $e^{-\alpha}$. Regarding Matveeva's experiment, ${ }^{12}$ she probably would have obtained much lower Ar concentrations at the anode had she reversed the electric field.

Tube Length: When $\delta$ and $\epsilon$ are kept constant, $\tau_{c}$ is also constant; thus $t_{c}$ is proportional to $L^{2}$. When $V_{o}$ and $V_{a}$ are kept constant, both $\delta$ and $\epsilon$ vary inversely with $L$; then $t_{c}$ is directly proportional to $L$. This prediction is in agreement with Matveeva ${ }^{12}$ and BGW. ${ }^{14}$

Because of the exponential dependence of $\alpha$, the tube length greatly influences the steady-state concentration profile. The longer the tube, the greater will be the concentration difference between the anode and cathode. This prediction is in agreement with experimental observations. ${ }^{11,12,14}$

Tube Radius: For fixed values of $V_{c}$ and $V_{a}, t_{c}$ is predicted to be proportional to $R^{-2}$. Unfortunately, there are no data available to test this prediction.

Concerning the steady-state concentration profile, changes in $R$ will influence $\alpha$. If the discharge current is kept constant, both the production rate of ions and the loss rate of ions should vary as $R^{-2}$; however, for small diameter tubes, $E$ decreases as $R$ increases., ${ }^{2,24}$ Thus, $\alpha$ should decrease with increasing $R$; this is in agreement with Schmeltekopf ${ }^{11}$ and with B GW. ${ }^{14}$

Pressure: Since the diffusion coefficient is inversely proportional to $P, t_{c}$ is predicted to be directly proportional to $P$; this is in agreement with Matveeva ${ }^{12}$ and B GW. ${ }^{14}$

Since both $\mu$ and $D$ are inversely proportional to $P$, $P$ affects $\alpha$ mainly through the ionizational fraction and through the electric field. Since the ambipolar diffusion coefficient is inversely proportional to $P$, the loss rate of ions should vary inversely with $P$. On the other hand,

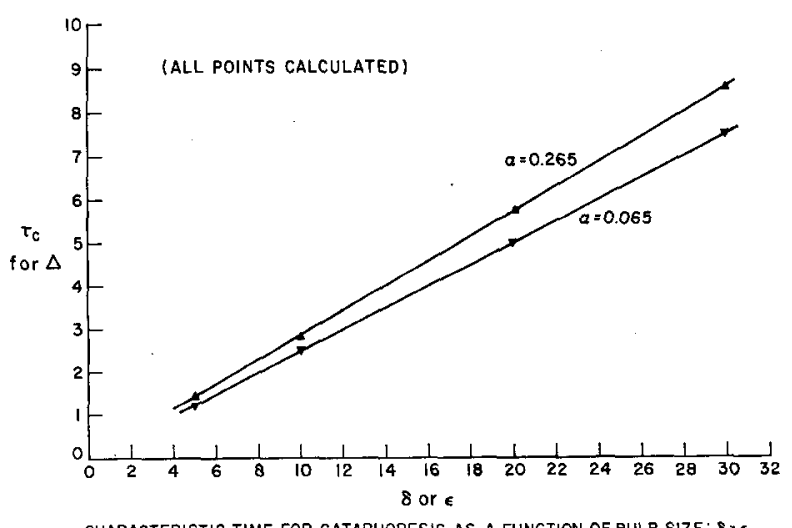

CHARACTERISTIC TIME FOR CATAPHORESIS AS A FUNCTION OF BULB SIZE: $8=\epsilon$

FIG. 6. Characteristic time for cataphoresis as a function of bulb size: $\delta=\varepsilon$. 
the production rate of ions may increase with increasing $P$. Both these effects enhance the ionization fraction and increase $\alpha$. The value of $\alpha$ may be further enhanced since the longitudinal electric field also increases with increasing pressure. ${ }^{24}$ The actual pressure dependence will depend upon the ionization mechanism. In the case of neon with argon impurity, the Penning effect helps keep the separation relatively pressure independent. ${ }^{18}$ Oskam ${ }^{18}$ also points out that in the case of helium with neon impurity, an increase in pressure will increase the production rate of $\mathrm{He}_{2}{ }^{+}$through the Hornbeck-Molnar process, $\mathrm{He}^{*}+\mathrm{He}_{-} \rightarrow \mathrm{He}_{2}{ }^{+}+e^{-}$, and through the three-body process $\mathrm{He}^{+}+2 \mathrm{He}^{+} \rightarrow \mathrm{He}_{2}{ }^{+}+\mathrm{He}$. These processes are important since the neon is probably ionized by $\mathrm{He}_{2}{ }^{+}+\mathrm{Ne} \rightarrow \mathrm{He}^{+}+2 \mathrm{He}$, as suggested by Loeb $^{15}$ and Oskam. ${ }^{18}$ At higher pressures, three-body recombination may become important thus tending to minimize the influence of pressure. Aside from the early work of Skaupy and Bobek, ${ }^{5}$ this is in agreement with experimental observations..$^{9,11,12,14}$

Discharge Current: If heating effects upon the transport properties are negligible, $t_{c}$ should not be significantly influenced by the discharge current; this is in agreement with experimental observations. ${ }^{12}$

Concerning the steady state, increasing the discharge current should increase $\alpha$ by increasing the ion production rate. As suggested in Schmeltekopf's empirical relation, ${ }^{11}$ the current density is more fundamental than the actual discharge current. Experimental observations have shown that the cataphoretic separation is enhanced with increasing discharge current..$^{5,9,11-14}$

Gas Temperature: The gas temperature is expected to influence $t_{c}$ by changing the diffusion coefficient.

Regarding the steady state, increasing the gas temperature increases the loss rate of ions to the wall

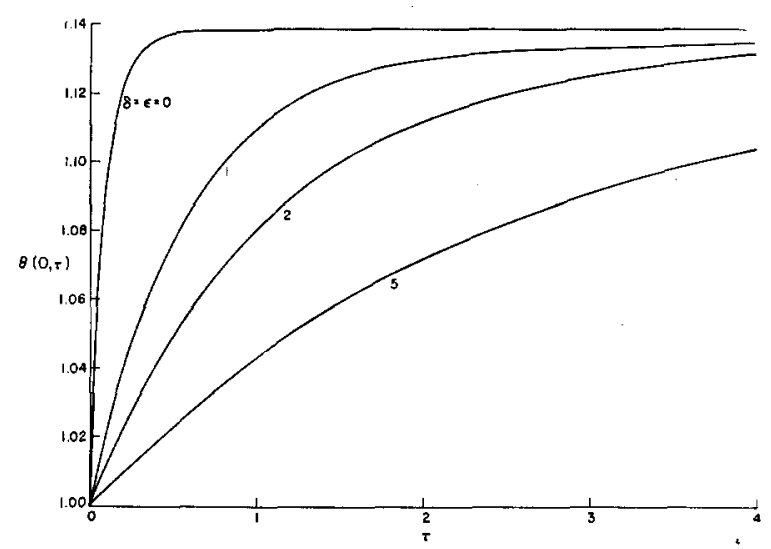

Fig. 7. Concentration at the cathode as a function of time for cataphoresis with equal size endbulbs $(\delta=\epsilon)$ for the case where $\alpha=0.265$.

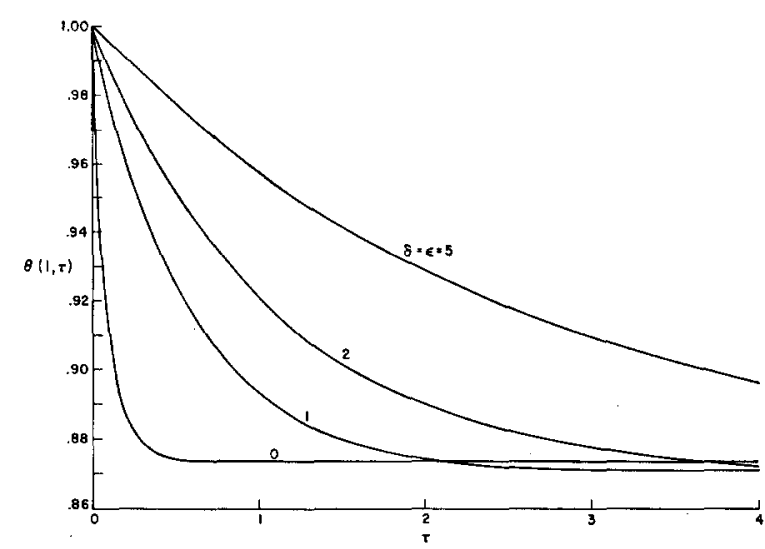

Fig. 8. Concentration at the anode as a function of time for cataphoresis with equal-size endbulbs $(\delta=\epsilon)$ for the case where $\alpha=0.265$.

and thus tends to reduce $\alpha$ by lowering the ionization fraction of the impurity. Experimental results ${ }^{11,14}$ indicate that the steady-state separation is reduced with increasing gas temperature.

Initial Composition: The model suggests that $t_{c}$ should be relatively independent of the initial composition of the impurity; this is in agreement with experiment ${ }^{12}$.

These experimental results also indicate that the steady-state separation increases with decreasing initial impurity concentration. Possibly, the ionization fraction of the impurity increases with decreasing concentration of impurity. If this be the case, the variation of $\alpha$ within each of the experiments analyzed was apparently weak enough to allow agreement between a linear model and an inherently nonlinear phenomenon. However, there are initial values of the impurity composition which will lead to a transition regime, where the ionization frequencies for the impurity gas and for the host gas are of the same order of magnitude. Then the electric field and the level of impurity ionization will be dependent upon the longitudinal position. ${ }^{30,31}$ In this case, variations from the linear model may be quite significant. Also, in some cases, the volume force in electrophoresis ${ }^{32}$ may give rise to deviations from the assumed model.

\section{ACKNOWLEDGMENT}

This work was supported in part by grants from the Sloan Foundation and the Shell Oil Company. Grateful acknowledgment is made to the donors of said funds.

${ }^{31}$ Yu A. Pekar, Sov. Phys. 12, 800 (1967).

${ }^{32}$ C. C. Leiby, Jr. and H. J. Oskam, Phys. Fluids 10, 1992 (1967). 